\title{
Vectorial dispersive shock waves on an incoherent landscape
}

\author{
Javier Nuño ${ }^{1,2}$, Christophe Finot ${ }^{1}$, Miro Erkintalo ${ }^{3,4}$, and Julien Fatome ${ }^{1,3,4, *}$ \\ ${ }^{1}$ Laboratoire Interdisciplinaire Carnot de Bourgogne, 9 av. A. Savary, Dijon, France \\ ${ }^{2}$ Departamento de Electrónica, Universidad de Alcalá, 28805 Alcalá de Henares (Madrid), Spain \\ ${ }^{3}$ Department of Physics, The University of Auckland, Private Bag 92019, Auckland 1142, New Zealand \\ ${ }^{4}$ The Dodd-Walls Centre for Photonic and Quantum Technologies, New Zealand
}

\begin{abstract}
We study the impact of temporal randomness on the formation of vectorial dispersive shock-waves that emerge due to the interaction of a partially coherent probe wave co-propagating together with an orthogonally polarized intense short pulse. Experiments carried out in a normally dispersive optical fiber demonstrate that the lack of coherence of the probe landscape acts as a strong diffusive term, which is able to hamper or inhibit the vectorial shock formation.
\end{abstract}

\section{Introduction}

In the last decades, shock waves have been the subject of intense studies in many areas of physics, ranging from hydrodynamics, nonlinear optics, condensates or plasmas [1]. Basically, shock waves occurring in conservative or low-dissipative systems originate from two fundamental ingredients: nonlinearity and dispersion. More specifically, in nonlinear optics, a self-defocusing regime of propagation can turn the front of an initial pulsed signal into a gradient catastrophe which is then regularized by chromatic dispersion. This regularization phenomenon then causes the onset of fast nonstationary oscillating fan structures called dispersive shock waves (DSWs) $[2,3]$ or undular bores [4] (in analogy with the classical "mascaret" which can be observed in specific river estuaries). Recently, we theoretically introduced and experimentally demonstrated a new type of vectorial DSW in optical fibers [5]. In contrast to previous studies, here a gradient catastrophe is generated on the phase of a $\mathrm{CW}$ probe through cross-phase modulation (XPM) induced by a pulsed signal owing to a vectorial configuration, leading to the equivalent of an optical axe [6]. We further explore here the nonlinear interaction occurring between the intense pulsed signal and the orthogonally polarized weak probe, especially in presence of partial incoherence. We provide here a set of experimental results carried at telecommunications wavelengths that have been closely confirmed by numerical simulations based on two coupled nonlinear Schrödinger equations corresponding to a simplified Manakov model [5].

\section{Results in the coherent case}

We first recall the basic principle of the vectorial interaction through XPM between a weak CW probe copropagating in an optical fiber together with an orthogonally polarized intense short pulse (supergaussian pulse with a duration of $68 \mathrm{ps}$ and a peak power of $3.8 \mathrm{~W}$ ). The temporal intensity profiles recorded after propagation in a $13-\mathrm{km}$ long normally dispersive fiber (dispersion of $-124 \mathrm{ps} / \mathrm{nm} / \mathrm{km}$ and nonlinear Kerr coefficient of $\left.5.2 \mathrm{~W}^{-1} \cdot \mathrm{km}^{-1}\right)$ are provided in Fig. 1. The defocusing regime induces a nonlinear reshaping of the pulsed signal into subsequently parabolic, then broad and expanding sharp square pulse until the wave breaking phenomenon occurs. This increasing sharpness and temporal expansion of the pulse edges then triggers an expanding catastrophe chirp profile on the orthogonally polarized $\mathrm{CW}$ probe through XPM coupling. Forced by this piston effect, this nonlinear phase gradient catastrophe is then regularized by chromatic dispersion, depleting the central region of the probe while creating two repulsive shock waves with oscillating fronts onto the initial CW landscape (Fig. 1b), thus mimicking superfluid or ballistic DSWs. Regarding the pulsed signal, it progressively becomes parabolic and finally propagates self-similarly, experiencing a strong temporal broadening (Fig. 1a).

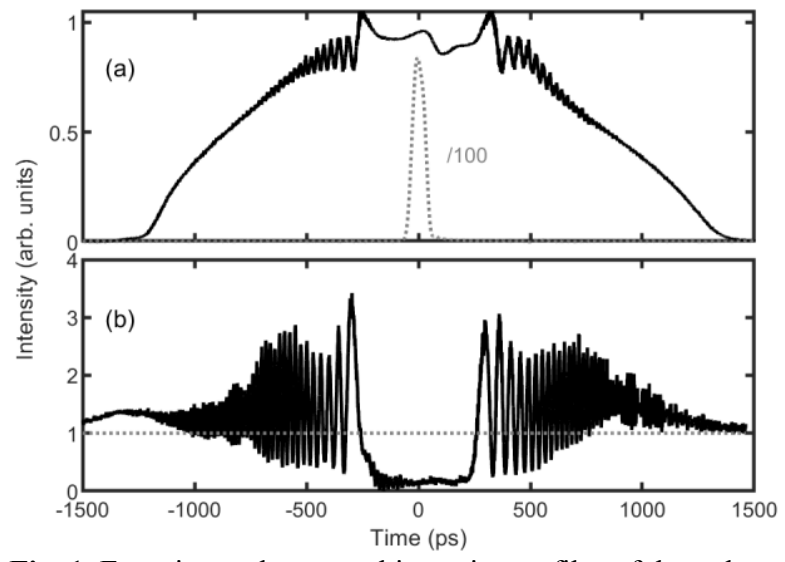

Fig. 1. Experimental temporal intensity profiles of the pulse wave (a) and of the continuous seed wave (b) at the input (dashed grey line) and output (solid black line) of the fiber.

\footnotetext{
$\overline{\text { * Corresponding author: cfinot@u-bourgogne.fr }}$
} 


\section{Vectorial shock wave on an incoherent landscape}

We replaced the coherent seed wave by the amplified spontaneous emission from an erbium-doped fiber amplifier. An optical bandpass filter with a tuneable spectral width is used to control the level of randomness of the probe. The 10-GHz temporal incoherent landscape after nonlinear propagation is visible in Fig. 2(a1) that results from the accumulation of a thousand of recorded traces. When the intense pulse is switched on, one can clearly see the typical temporal gap emerging from the fluctuations of the $10 \mathrm{GHz}$ partially coherent wave. We can also make out the existence of bumps localized on each side of the temporal gap, typical of this vectorial DSW dynamics. The details of the fan structure are however completely blurred by the incoherence of the probe. Moreover, details of the deterministic pulse reported in panel (a2) show that the level of fluctuations may strongly vary along the pulse width. Indeed, significant intensity fluctuations surrounding the central part of the pulse are observed, confirming once again the XPM induced by the incoherent probe wave around the rarefaction area. In contrast, the central part and the wings of the parabolic profile remain fully coherent.

In order to further assess the statistical behavior of the waves, we have also experimentally evaluated the probability distribution function of the intensity profiles along two different temporal areas of the waves. Results are summarized in panels (b1) and (b2) for the probe and pulse waves, respectively. Whereas the rarefaction area of the probe (open blue circles, blue region in panel (a)) presents a narrow distribution centered close to zero, the bumps (red full circles, red regions in panel (a) are characterized by temporal events presenting higher peak power than the ones appearing without any interaction with the pulse (yellow crosses, yellow region in panel (a)). Regarding the pulse, the experiments confirm that the pdf of the central part (rarefaction area of the probe) is much narrower than the ones affected by the DSWs fluctuations (on the edge of the temporal gap).

Finally, we investigate in panel (c) the impact of the degree of incoherence in the probe landscape by monitoring the temporally averaged DSWs by means of a 70-GHz sampling oscilloscope triggered by means of the output pulse. For a 10- or 20-GHz incoherent landscape, we can still observe the piston effect caused by the pulse induced XPM on the probe signal and in particular the depletion of the central part. However, the fast temporal DSW fan structures tend to be damped by the randomness of the landscape. Further increasing the amount of disorder then tends to even more hamper the shock wave until its total inhibition for an incoherence higher than 40 GHz. For an incoherence of $100 \mathrm{GHz}$, the averaged recording only presents a small bump All the statistical trends observed experimentally have been closely confirmed by intensive numerical simulations of the Manakov model.
1. G. B. Whitham, Linear and Nonlinear Waves (Wiley, New York, 1974).

2. G. Xu, M. Conforti, A. Kudlinski, A. Mussot, and S. Trillo, Phys. Rev. Lett. 118, 254101 (2017).

3. G. A. El and M. A. Hoefer, "Dispersive shock waves and modulation theory," Physica D 333, 11-65 (2016).

4. J. Fatome, C. Finot, G. Millot, A. Armaroli, and S. Trillo, Phys. Rev. X 4, 021022 (2014).

5. J. Nuno, C. Finot, G. Xu, G. Millot, M. Erkintalo, and J. Fatome, Commun. Phys. 2, 138 (2019).

6. L. Helczynski, B. Hall, D. Anderson, M. Lisak, A. Berntson, and M. Desaix, Phys. Scr. T84, 81 (2000).
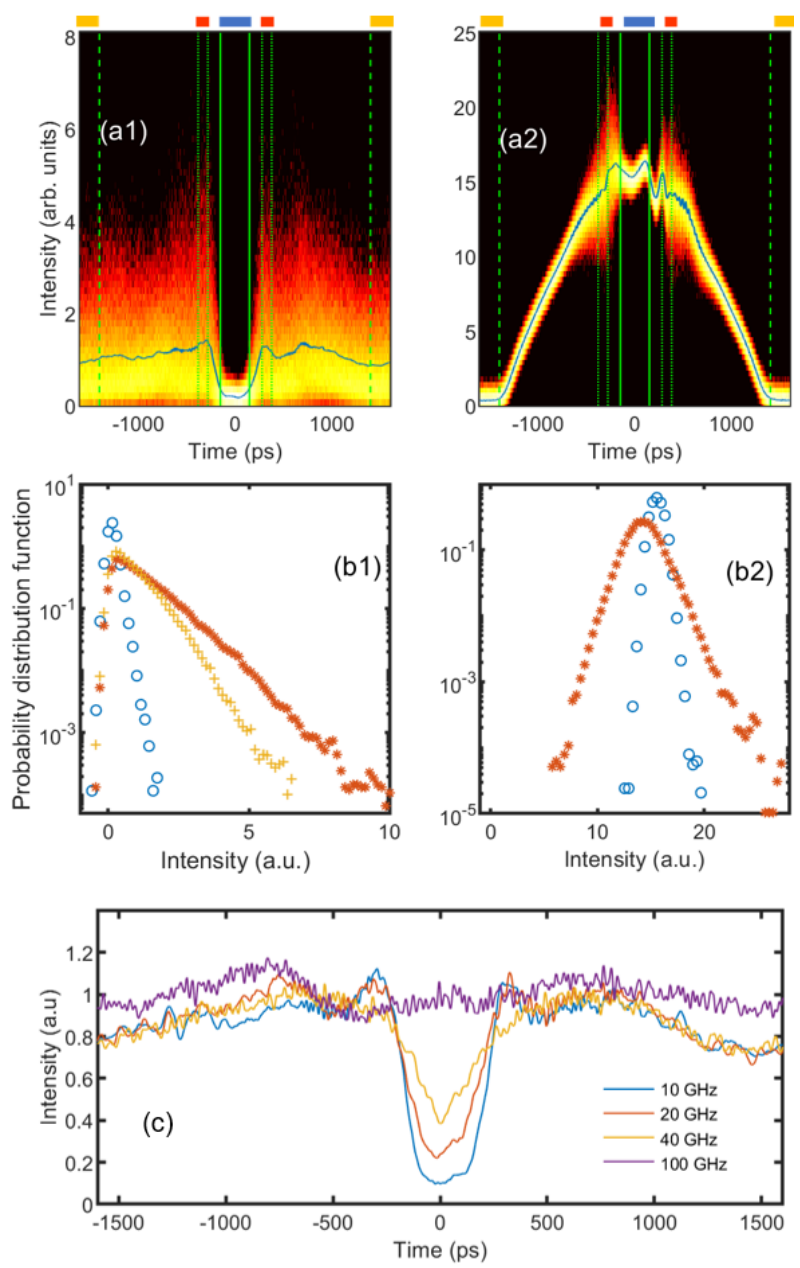

Fig. 2. Impact of the landscape partial incoherence on the vectorial DSWs. (a-b) Experimental results obtained for a pulse -peak power of $3.8 \mathrm{~W}$ and a spectral coherence of the probe of $10 \mathrm{GHz}$. (a) Output intensity profiles recorded for the probe and pulse waves are plotted in panels (a1) and (a2) respectively. (b) Evolution of the pdf in two different temporal sections of the probe and pulse waves (panel b1 and b2 respectively). Statistics for the central part of the probe (blue part in panels (a)) are plotted with blue open circles whereas properties in the lateral bumps (red parts in panels (a)) are plotted with red stars and with yellow crosses for the region outside the temporal pulse extend (yellow part in panels (a)). (c) Impact of the partial incoherence on the probe signal. Output temporal intensity profile of the probe wave after averaging over 1000 shots. Different levels of initial incoherence are compared: 10, 20, 40 and $100 \mathrm{GHz}$ (blue, red, yellow and purple colors, respectively). 